\title{
CONVERGENCE RATE OF EM SCHEME FOR SDDEs
}

\author{
JIANHAI BAO AND CHENGGUI YUAN
}

(Communicated by Edward C. Waymire)

\begin{abstract}
In this paper we investigate the convergence rate of the EulerMaruyama (EM) scheme for a class of stochastic differential delay equations, where the corresponding coefficients may be highly nonlinear with respect to the delay variables. In particular, we reveal that the convergence rate of the Euler-Maruyama scheme is $\frac{1}{2}$ for the Brownian motion case, while we show that it is best to use the mean-square convergence for the pure-jump case and that the order of mean-square convergence is close to $\frac{1}{2}$.
\end{abstract}

\section{INTRODUCTION}

Since most stochastic differential equations (SDEs) cannot be solved explicitly, numerical methods have become essential. Recently, there has been extensive literature in investigating the strong convergence, weak convergence or sample path convergence of numerical schemes for SDEs, e.g., in [3] for SDEs with a monotone condition, in [4, 7, 12, for SDEs with jumps, in [6, 7, 9, 10] for stochastic differential delay equations (SDDEs), and in 4, 5, for SDEs with a one-side Lipschitz condition. For the comprehensive monographs on numerical approximate methods of SDEs, we refer to, e.g., [8, 12, 13. Although the results on the convergence of Euler-Maruyama (EM) schemes are substantial, there are limited results on the convergence rate under weaker conditions than the global Lipschitz condition and the linear growth condition. For example, a recent work in 2 reveals the convergence rate of the EM scheme for a class of SDEs under a Hölder condition, and, with local Lipschitz constants satisfying logarithm growth conditions, [15] and [1, 7] discuss the convergence rate of EM approximate methods for SDEs and stochastic functional differential equations with jumps, respectively. We should also point out that the strong convergence of EM schemes for SDDEs is, in general, discussed under a linear growth condition or bounded moments of analytic and numerical solutions, e.g., [7, 9, 10], and that the convergence rate [1, 7] is also revealed under a linear growth condition.

Received by the editors November 17, 2011.

2010 Mathematics Subject Classification. Primary 65C30; Secondary 60H10, 65L20.

Key words and phrases. Stochastic differential delay equation, highly nonlinear, jumps, EM scheme, convergence rate. 
To further motivate our work, we first consider an SDDE on $\mathbb{R}$,

$$
d X(t)=\left\{a X(t)+b X^{3}(t-\tau)\right\} d t+c X^{2}(t-\tau) d W(t),
$$

where $a, b, c \in \mathbb{R}, \tau>0$ are constant and $W(t)$ is a scalar Brownian motion. It is easy to observe that both the drift coefficient and the diffusion coefficient are highly nonlinear, especially with respect to the delay arguments. Therefore, the existing convergence results, e.g., [7, 9, 10, cannot cover (1.1), and the convergence rate of the corresponding EM scheme also cannot be revealed by the techniques of [1, 7 ] as we explained at the end of the last paragraph. On the other hand, our work is also enlightened by the recent work [2] due to Gyöngy and Rásonyi. In [2] they considered SDE on $\mathbb{R}$,

$$
d X(t)=\{f(t, X(t))+g(t, X(t))\} d t+\sigma(t, X(t)) d W(t),
$$

and discussed the convergence rate of the associated EM method, where $g$ is Hölder continuous, of linear growth, and monotone decreasing with respect to the second variable.

Motivated by the previous literature, in this paper we shall not only study the strong convergence of EM schemes for a class of SDDEs, which may be highly nonlinear with respect to the delay variables, but also reveal the convergence rate of the corresponding EM numerical methods. The rest of the paper is organized as follows: under highly nonlinear growth conditions with respect to the delay arguments, in Section 2 we reveal that the convergence rate of EM schemes for SDDEs driven by Brownian motion is $\frac{1}{2}$, while in Section 3 we show that it is best to use the mean-square convergence for the pure jump case and verify that the rate of mean-square convergence is close to $\frac{1}{2}$.

\section{Convergence Rate for the Brownian motion CASE}

For an integer $n>0$, let $\left(\mathbb{R}^{n},\langle\cdot, \cdot\rangle,|\cdot|\right)$ be the Euclidean space and $\|A\|:=$ $\sqrt{\operatorname{trace}\left(A^{*} A\right)}$ the Hilbert-Schmidt norm for a matrix $A$, where $A^{*}$ is its transpose. Let $W(t)$ be an $m$-dimensional Brownian motion defined on some complete probability space $\left(\Omega, \mathcal{F}, \mathbb{P},\left\{\mathcal{F}_{t}\right\}_{t \geq 0}\right)$. Throughout the paper, $C>0$ denotes a generic constant whose values may change from line to line.

For fixed $T>0$, in this section we consider SDDE on $\mathbb{R}^{n}$ :

$$
d X(t)=b(X(t), X(t-\tau)) d t+\sigma(X(t), X(t-\tau)) d W(t), \quad t \in[0, T],
$$

with initial data $X(\theta)=\xi(\theta), \theta \in[-\tau, 0]$.

To guarantee the existence and uniqueness of solutions we introduce the following conditions. Let $V_{i}: \mathbb{R}^{n} \times \mathbb{R}^{n} \rightarrow \mathbb{R}_{+}$such that

$$
V_{i}(x, y) \leq K_{i}\left(1+|x|^{q_{i}}+|y|^{q_{i}}\right), \quad i=1,2,
$$

for some $K_{i}>0, q_{i} \geq 1$ and arbitrary $x, y \in \mathbb{R}^{n}$. We further assume that

(A1) $b: \mathbb{R}^{n} \times \mathbb{R}^{n} \rightarrow \mathbb{R}^{n}$ and there exists $L_{1}>0$ such that

$$
\left|b\left(x_{1}, y_{1}\right)-b\left(x_{2}, y_{2}\right)\right| \leq L_{1}\left|x_{1}-x_{2}\right|+V_{1}\left(y_{1}, y_{2}\right)\left|y_{1}-y_{2}\right|
$$

for $x_{i}, y_{i} \in \mathbb{R}^{n}, i=1,2$;

(A2) $\sigma: \mathbb{R}^{n} \times \mathbb{R}^{n} \rightarrow \mathbb{R}^{n \times m}$ and there exists $L_{2}>0$ such that

$$
\left\|\sigma\left(x_{1}, y_{1}\right)-\sigma\left(x_{2}, y_{2}\right)\right\| \leq L_{2}\left|x_{1}-x_{2}\right|+V_{2}\left(y_{1}, y_{2}\right)\left|y_{1}-y_{2}\right|
$$

for $x_{i}, y_{i} \in \mathbb{R}^{n}, i=1,2$. 
We now introduce an EM method for (2.1). Without loss of generality, we may assume that there exist sufficiently large integers $N, M>0$ such that

$$
\triangle:=\frac{\tau}{N}=\frac{T}{M} \in(0,1) .
$$

Define a continuous EM scheme associated with (2.1):

$$
d Y(t)=b(\bar{Y}(t), \bar{Y}(t-\tau)) d t+\sigma(\bar{Y}(t), \bar{Y}(t-\tau)) d W(t)
$$

where $\bar{Y}(t):=Y(k \triangle)$ for $t \in[k \triangle,(k+1) \triangle), k=0,1, \cdots, M-1$, and $\bar{Y}(\theta):=$ $\xi(\theta), \theta \in[-\tau, 0]$.

Remark 2.1. Clearly, if $b$ and $\sigma$ are globally Lipschitzian, then $b$ and $\sigma$ satisfy (A1)(A2). On the other hand, we remark that $b$ and $\sigma$ may be highly nonlinear with respect to the delay variables. There are many examples which are covered by (A1)(A2). For example, for (1.1) it is trivial to see that $b(x, y)=a x+b y^{3}, \sigma(x, y)=c y^{2}$, and (A1)-(A2) hold by choosing $V_{1}(x, y)=\frac{3|b|}{2}\left(x^{2}+y^{2}\right)$ and $V_{2}(x, y)=|c|(|x|+|y|)$, respectively. In fact, the examples where the drift coefficient and the diffusion coefficient are polynomial of degree $d \geq 1$ with regard to the delay variables are also included in our framework.

Lemma 2.2. Assume that (A1) and (A2) hold. Then, for any initial data $\xi \in$ $C_{\mathcal{F}_{0}}^{b}\left([-\tau, 0] ; \mathbb{R}^{n}\right),(2.1)$ admits a unique global strong solution $X(t), t \in[0, T]$. Moreover, for any $p \geq 2$ there exists $C>0$ independent of $\triangle$ such that

$$
\mathbb{E}\left(\sup _{0 \leq t \leq T}|X(t)|^{p}\right) \vee \mathbb{E}\left(\sup _{0 \leq t \leq T}|Y(t)|^{p}\right) \leq C
$$

and

$$
\mathbb{E}|Y(t)-\bar{Y}(t)|^{p} \leq C \triangle^{\frac{p}{2}}
$$

Proof. Note that (2.1) has a unique local solution due to the fact that both $b$ and $\sigma$ are locally Lipschitzian. To verify that (2.1) admits a unique global solution on time interval $[0, T]$, it is sufficient to show that

$$
\mathbb{E}\left(\sup _{0 \leq t \leq T}|X(t)|^{p}\right) \leq C, \quad p \geq 2
$$

By a straightforward computation, we deduce from (A1), (A2) and (2.2) that

$$
|b(x, y)| \leq C\left(1+|x|+|y|+|y|^{q_{1}+1}\right), \quad x, y \in \mathbb{R}^{n},
$$

and

$$
\|\sigma(x, y)\| \leq C\left(1+|x|+|y|+|y|^{q_{2}+1}\right), \quad x, y \in \mathbb{R}^{n} .
$$

Set $\gamma_{1}:=q_{1}+1$ and $\gamma_{2}:=q_{2}+1$. To show (2.7), by (2.8), (2.9), the Hölder inequality and the Burkhold-Davis-Gundy inequality, we obtain that for any $p \geq 2$ 
and $t \in[0, T]$,

$$
\begin{aligned}
\mathbb{E}\left(\sup _{0 \leq s \leq t}|X(s)|^{p}\right) \leq & 3^{p-1}\left\{|\xi(0)|^{p}+\mathbb{E}\left(\sup _{0 \leq s \leq t}\left|\int_{0}^{s} b(X(r), X(r-\tau)) d r\right|^{p}\right)\right. \\
& \left.+\mathbb{E}\left(\sup _{0 \leq s \leq t}\left|\int_{0}^{s} \sigma(X(r), X(r-\tau)) d W(r)\right|^{p}\right)\right\} \\
\leq & C\left\{1+\mathbb{E} \int_{0}^{t}\left(|b(X(s), X(s-\tau))|^{p}+\|\sigma(X(s), X(s-\tau))\|^{p}\right) d s\right\} \\
\leq & C\left\{1+\mathbb{E} \int_{0}^{t}|X(s)|^{p} d s+\mathbb{E} \int_{0}^{t}\left(|X(s-\tau)|^{p \gamma_{1}}+|X(s-\tau)|^{p \gamma_{2}}\right) d s\right\},
\end{aligned}
$$

where we have also used the Young inequality in the last step. This, together with the Gronwall inequality, yields that for $t \in[0, T]$ and $p \geq 2$,

$$
\mathbb{E}\left(\sup _{0 \leq s \leq t}|X(s)|^{p}\right) \leq C\left\{1+\mathbb{E} \int_{0}^{t}\left(|X(s-\tau)|^{p \gamma_{1}}+|X(s-\tau)|^{p \gamma_{2}}\right) d s\right\} .
$$

The following argument is similar to that of [14, Theorem 2.1], while we give a detailed proof since it is helpful to show the convergence rate (see the proof of Theorem 2.4). Let $\beta:=\gamma_{1} \vee \gamma_{2}$ and

$$
p_{i}:=([T / \tau]+2-i) p \beta^{[T / \tau]+1-i}, \quad i=1,2, \cdots,[T / \tau]+1,
$$

where $[a]$ denotes the integer part of the real number $a$. Thus, due to $\beta \geq 1$ and $p \geq 2$, it is easy to see that $p_{i} \geq 2$ such that

$$
p_{i+1} \beta<p_{i} \text { and } p_{[T / \tau]+1}=p, \quad i=1,2, \cdots,[T / \tau] .
$$

By (2.10), together with $\xi \in C_{\mathcal{F}_{0}}^{b}\left([-\tau, 0] ; \mathbb{R}^{n}\right)$, we obtain that

$$
\mathbb{E}\left(\sup _{0 \leq s \leq \tau}|X(s)|^{p_{1}}\right) \leq C .
$$

Combining (2.10) with the Hölder inequality further leads to

$$
\begin{aligned}
\mathbb{E}\left(\sup _{0 \leq s \leq 2 \tau}|X(s)|^{p_{2}}\right) & \leq C\left\{1+\mathbb{E} \int_{0}^{2 \tau}\left(|X(s-\tau)|^{p_{2} \gamma_{1}}+|X(s-\tau)|^{p_{2} \gamma_{2}}\right) d s\right\} \\
& \leq C\left\{1+\int_{0}^{\tau}\left(\left(\mathbb{E}|X(s)|^{p_{1}}\right)^{\frac{p_{2} \gamma_{1}}{p_{1}}}+\left(\mathbb{E}|X(s)|^{p_{1}}\right)^{\frac{p_{2} \gamma_{2}}{p_{1}}}\right) d s\right\} \\
& \leq C .
\end{aligned}
$$

Repeating the previous procedures gives (2.7) and $\mathbb{E}\left(\sup _{0 \leq t \leq T}|Y(t)|^{p}\right) \leq C$. Finally, statement (2.6) can also be obtained by taking the Hölder inequality, the Burkhold-Davis-Gundy inequality and (2.5) into account.

Remark 2.3. Lemma 2.2 gives a new result on the existence and uniqueness of solutions to SDDEs on the finite-time interval, where the coefficients may be polynomial of any degree $d \geq 1$ with regard to the delay variables.

We now state our main result, which not only shows the strong convergence of EM scheme associated with (2.1) but also reveals its convergence rate, although the drift coefficient and the diffusion coefficient may be highly nonlinear with respect to the delay arguments. 
Theorem 2.4. Under (A1) and (A2), for any $p \geq 2$ there exists $C>0$ such that

$$
\mathbb{E}\left(\sup _{0 \leq s \leq T}|X(s)-Y(s)|^{p}\right) \leq C \triangle^{\frac{p}{2}}
$$

that is, the rate of convergence of the EM scheme (2.4) is $\frac{1}{2}$.

Proof. The argument is motivated by that of [2, Theorem 2.1]. For fixed $\delta>1$ and arbitrary $\epsilon \in(0,1)$, there exists a continuous nonnegative function $\psi_{\delta \epsilon}(x), x \geq 0$, with support $[\epsilon / \delta, \epsilon]$, such that

$$
\int_{\epsilon / \delta}^{\epsilon} \psi_{\delta \epsilon}(x) d x=1 \text { and } \psi_{\delta \epsilon}(x) \leq \frac{2}{x \ln \delta}, \quad x>0 .
$$

Define

$$
\phi_{\delta \epsilon}(x):=\int_{0}^{x} \int_{0}^{y} \psi_{\delta \epsilon}(z) d z d y, \quad x>0 .
$$

Then $\phi_{\delta \epsilon} \in C^{2}\left(\mathbb{R}_{+} ; \mathbb{R}_{+}\right)$possesses the following properties:

$$
x-\epsilon \leq \phi_{\delta \epsilon}(x) \leq x, \quad x>0,
$$

and

$$
0 \leq \phi_{\delta \epsilon}^{\prime}(x) \leq 1, \quad \phi_{\delta \epsilon}^{\prime \prime}(x) \leq \frac{2}{x \ln \delta} \mathbf{1}_{[\epsilon / \delta, \epsilon]}(x), \quad x>0
$$

Define

$$
V_{\delta \epsilon}(x):=\phi_{\delta \epsilon}(|x|), \quad x \in \mathbb{R}^{n} .
$$

By the definition of $\phi_{\delta \epsilon}$, it is trivial to note that $V_{\delta \epsilon} \in C^{2}\left(\mathbb{R}^{n} ; \mathbb{R}_{+}\right)$. Set

$$
\left(V_{\delta \epsilon}\right)_{x}(x):=\left(\frac{\partial V_{\delta \epsilon}(x)}{\partial x_{1}}, \cdots, \frac{\partial V_{\delta \epsilon}(x)}{\partial x_{n}}\right) \text { and }\left(V_{\delta \epsilon}\right)_{x x}(x):=\left(\frac{\partial^{2} V_{\delta \epsilon}(x)}{\partial x_{i} \partial x_{j}}\right)_{n \times n}, \quad x \in \mathbb{R}^{n} .
$$

By a straightforward computation, we deduce that

$$
\frac{\partial V_{\delta \epsilon}(x)}{\partial x_{i}}=\phi_{\delta \epsilon}^{\prime}(|x|) \frac{x_{i}}{|x|}
$$

and

$$
\frac{\partial^{2} V_{\delta \epsilon}(x)}{\partial x_{i} \partial x_{j}}=\phi_{\delta \epsilon}^{\prime}(|x|)\left(\delta_{i j}|x|^{2}-x_{i} x_{j}\right)|x|^{-3}+\phi_{\delta \epsilon}^{\prime \prime}(|x|) x_{i} x_{j}|x|^{-2},
$$

for $x \in \mathbb{R}^{n}$ and $i=1,2, \cdots, n$, where $\delta_{i j}=1$ if $i=j$ or otherwise 0 , and then that

$$
0 \leq\left|\left(V_{\delta \epsilon}\right)_{x}(x)\right| \leq 1 \text { and }\left\|\left(V_{\delta \epsilon}\right)_{x x}(x)\right\| \leq 2 n\left(1+\frac{1}{\ln \delta}\right) \frac{1}{|x|} \mathbf{1}_{[\epsilon / \delta, \epsilon]}(|x|)
$$

for $x \in \mathbb{R}^{n}$. For any $t \in[0, T]$, let

$$
Z(t):=X(t)-Y(t), \quad \bar{Z}(t):=Y(t)-\bar{Y}(t) \text { and } \tilde{Z}(t):=(X(t), \bar{Y}(t)) \in \mathbb{R}^{2 n} .
$$


Applying the Itô formula yields that

$$
\begin{aligned}
V_{\delta \epsilon}(Z(t))= & \int_{0}^{t}\left\langle\left(V_{\delta \epsilon}\right)_{x}(Z(s)), b(X(s), X(s-\tau))-b(\bar{Y}(s), \bar{Y}(s-\tau))\right\rangle d s \\
& +\frac{1}{2} \int_{0}^{t} \operatorname{trace}\left\{(\sigma(X(s), X(s-\tau))-\sigma(\bar{Y}(s), \bar{Y}(s-\tau)))^{*}\left(V_{\delta \epsilon}\right)_{x x}(Z(s))\right. \\
& \times(\sigma(X(s), X(s-\tau))-\sigma(\bar{Y}(s), \bar{Y}(s-\tau)))\} d s \\
& +\int_{0}^{t}\left\langle\left(V_{\delta \epsilon}\right)_{x}(Z(s)),(\sigma(X(s), X(s-\tau))-\sigma(\bar{Y}(s), \bar{Y}(s-\tau))) d W(s)\right\rangle \\
= & : I_{1}(t)+I_{2}(t)+I_{3}(t) .
\end{aligned}
$$

By (2.14), (A1) and the Hölder inequality, we derive that

$$
\begin{aligned}
\mathbb{E}\left(\sup _{0 \leq s \leq t}\left|I_{1}(s)\right|^{p}\right) \leq & \int_{0}^{t} \mathbb{E}|b(X(s), X(s-\tau))-b(\bar{Y}(s), \bar{Y}(s-\tau))|^{p} d s \\
\leq & C \int_{0}^{t}\left\{\mathbb{E}|Z(s)|^{p}+\mathbb{E}|\bar{Z}(s)|^{p}\right. \\
& +\left(\mathbb{E} V_{1}^{2 p}(\tilde{Z}(s-\tau))\right)^{\frac{1}{2}}\left(\mathbb{E}|Z(s-\tau)|^{2 p}\right)^{\frac{1}{2}} \\
& \left.+\left(\mathbb{E} V_{1}^{2 p}(\tilde{Z}(s))\right)^{\frac{1}{2}}\left(\mathbb{E}|\bar{Z}(s)|^{2 p}\right)^{\frac{1}{2}}\right\} d s, \quad t \in[0, T]
\end{aligned}
$$

and due to (A2) and (2.14) again that

$$
\begin{aligned}
\mathbb{E}\left(\sup _{0 \leq s \leq t}\left|I_{2}(s)\right|^{p}\right) \leq & C \int_{0}^{t} \mathbb{E}\left\{\left\|\left(V_{\delta \epsilon}\right)_{x x}(Z(s))\right\|\right. \\
& \left.\times\|\sigma(X(s), X(s-\tau))-\sigma(\bar{Y}(s), \bar{Y}(s-\tau))\|^{2}\right\}^{p} d s \\
\leq & C \mathbb{E} \int_{0}^{t} \frac{1}{|Z(s)|^{p}}\left\{|Z(s)|^{2 p}+|\bar{Z}(s)|^{2 p}\right. \\
& +V_{2}^{2 p}(\tilde{Z}(s-\tau))|Z(s-\tau)|^{2 p} \\
& \left.+V_{2}^{2 p}(\tilde{Z}(s-\tau))|\bar{Z}(s-\tau)|^{2 p}\right\} \mathbf{1}_{[\epsilon / \delta, \epsilon]}(|Z(s)|) d s \\
\leq & C \int_{0}^{t}\left\{\mathbb{E}|Z(s)|^{p}+\frac{1}{\epsilon^{p}} \mathbb{E}|\bar{Z}(s)|^{2 p}\right. \\
& +\frac{1}{\epsilon^{p}}\left(\mathbb{E} V_{2}^{4 p}(\tilde{Z}(s-\tau))\right)^{\frac{1}{2}}\left(\mathbb{E}|Z(s-\tau)|^{4 p}\right)^{\frac{1}{2}} \\
& \left.+\frac{1}{\epsilon^{p}}\left(\mathbb{E} V_{2}^{4 p}(\tilde{Z}(s))\right)^{\frac{1}{2}}\left(\mathbb{E}|\bar{Z}(s)|^{4 p}\right)^{\frac{1}{2}}\right\} d s .
\end{aligned}
$$


By virtue of the Burkhold-Davis-Gundy inequality, the Hölder inequality and (2.14), for any $p \geq 2$ and $t \in[0, T]$,

$$
\begin{aligned}
\mathbb{E}\left(\sup _{0 \leq s \leq t}\left|I_{3}(s)\right|^{p}\right) \leq & C \mathbb{E} \int_{0}^{t}\|\sigma(X(s), X(s-\tau))-\sigma(\bar{Y}(s), \bar{Y}(s-\tau))\|^{p} d s \\
\leq & C \int_{0}^{t}\left\{\mathbb{E}|Z(s)|^{p} d s+\mathbb{E}|\bar{Z}(s)|^{p}\right. \\
& +\left(\mathbb{E} V_{2}^{2 p}(\tilde{Z}(s-\tau))\right)^{\frac{1}{2}}\left(\mathbb{E}|Z(s-\tau)|^{2 p}\right)^{\frac{1}{2}} \\
& \left.+\left(\mathbb{E} V_{2}^{2 p}(\tilde{Z}(s))\right)^{\frac{1}{2}}\left(\mathbb{E}|\bar{Z}(s)|^{2 p}\right)^{\frac{1}{2}}\right\} d s .
\end{aligned}
$$

Furthermore, observe from (2.2) and (2.5) that

$$
\mathbb{E} V_{1}^{2 p}(\tilde{Z}(s-\tau))+\mathbb{E} V_{2}^{4 p}(\tilde{Z}(s-\tau)) \leq C
$$

and that $\mathbb{E}|\bar{Z}(t)|^{p} \leq C \triangle^{\frac{p}{2}}$ by (2.6). Then, combining (2.15) and (2.16) with (2.17), we obtain from (2.11) that for any $t \in[0, T]$ and any $p \geq 2$,

$$
\begin{aligned}
\mathbb{E}\left(\sup _{0 \leq s \leq t}|Z(s)|^{p}\right) \leq & 2^{p-1}\left\{\epsilon^{p}+\mathbb{E}\left(\sup _{0 \leq s \leq t} V_{\delta \epsilon}^{p}(Z(s))\right)\right\} \\
\leq & C\left\{\epsilon^{p}+\triangle^{\frac{p}{2}}+\frac{1}{\epsilon^{p}} \triangle^{p}+\frac{1}{\epsilon^{p}} \triangle^{p}\right. \\
& +\int_{0}^{t} \mathbb{E}|Z(s)|^{p} d s+\int_{0}^{t}\left(\mathbb{E}|Z(s-\tau)|^{2 p}\right)^{\frac{1}{2}} d s \\
& \left.+\frac{1}{\epsilon^{p}} \int_{0}^{t}\left(\mathbb{E}|Z(s-\tau)|^{4 p}\right)^{\frac{1}{2}} d s\right\} .
\end{aligned}
$$

This, together with the Gronwall inequality, further implies that

$$
\begin{aligned}
\mathbb{E}\left(\sup _{0 \leq s \leq t}|Z(s)|^{p}\right) \leq & C\left\{\epsilon^{p}+\triangle^{\frac{p}{2}}+\frac{1}{\epsilon^{p}} \triangle^{p}+\int_{0}^{t}\left(\mathbb{E}|Z(s-\tau)|^{2 p}\right)^{\frac{1}{2}} d s\right. \\
& \left.+\frac{1}{\epsilon^{p}} \int_{0}^{t}\left(\mathbb{E}|Z(s-\tau)|^{4 p}\right)^{\frac{1}{2}} d s\right\} .
\end{aligned}
$$

For any $p \geq 2$, let

$$
p_{i}:=([T / \tau]+2-i) p 4^{[T / \tau]+1-i}, \quad i=1,2, \cdots,[T / \tau]+1 .
$$

It is easy to see that

$$
4 p_{i+1}<p_{i} \text { and } p_{[T / \tau]+1}=p, \quad i=1,2, \cdots,[T / \tau] .
$$

Noting that $Z(s-\tau)=0$ for $s \in[0, \tau]$ and taking $\epsilon=\triangle^{\frac{1}{2}}$ in (2.18), we obtain that

$$
\mathbb{E}\left(\sup _{0 \leq s \leq \tau}|Z(s)|^{p_{1}}\right) \leq C \triangle^{\frac{p 1}{2}} .
$$

This, together with (2.19) and the Hölder inequality, further gives that

$$
\begin{aligned}
\mathbb{E}\left(\sup _{0 \leq s \leq 2 \tau}|Z(s)|^{p_{2}}\right) \leq & C\left\{\triangle^{\frac{p_{2}}{2}}+\int_{0}^{2 \tau}\left(\mathbb{E}|Z(s-\tau)|^{p_{1}}\right)^{\frac{p_{2}}{p_{1}}} d s\right. \\
& \left.+\triangle^{-\frac{p_{2}}{2}} \int_{0}^{2 \tau}\left(\mathbb{E}|Z(s-\tau)|^{p_{1}}\right)^{\frac{2 p_{2}}{p_{1}}} d s\right\} \\
\leq & C \triangle^{\frac{p 2}{2}}
\end{aligned}
$$


by taking $\epsilon=\triangle^{\frac{1}{2}}$ in (2.18). The desired assertion then follows by repeating the previous procedures.

Remark 2.5. The strong convergence of the EM scheme for SDDEs is generally investigated under the local Lipschitz condition and bounded moments of analytic solutions and numerical solutions or under the local Lipschitz condition and linear growth condition; see, e.g., [10. In this section, for a class of SDDEs which may be highly nonlinear with respect to the delay variables, we show the strong convergence of the EM scheme under rather general conditions. To the best of our knowledge, there are relatively few results in the existing literature.

Remark 2.6. There are only limited results on the convergence order of the EM scheme for SDEs or SDDEs under weaker conditions than the global Lipschitz and linear growth conditions. For example, under a Hölder continuous condition, 2 revealed the convergence order of the EM scheme for a class of SDEs with Hölder continuity, and, with local Lipschitz constants satisfying logarithm growth conditions, [15] and [1, 7] discussed the convergence rate of the EM approximate methods for SDEs and stochastic functional differential equations with jumps respectively, where linear growth condition is imposed in [1, 7]. In this section, under very general conditions, we reveal the convergence order of the EM scheme for a class of SDDEs, although they are highly nonlinear with respect to delay arguments.

\section{Convergence Rate for the pure Jump CASE}

In this last section we discuss the strong convergence of the EM scheme for a class of SDDEs and reveal that the convergence rate is $\frac{1}{2}$, although both the drift coefficient and the diffusion coefficient may be highly nonlinear with respect to the delay variables. In this section we turn to the counterpart for SDDEs with jumps. We further need to introduce some notation. Let $\mathcal{B}(\mathbb{R})$ be the Borel $\sigma$ algebra on $\mathbb{R}$ and $\lambda(d x)$ be a $\sigma$-finite measure defined on $\mathcal{B}(\mathbb{R})$. Let $p=(p(t)), t \in$ $D_{p}$, be a stationary $\mathcal{F}_{t}$-Poisson point process on $\mathbb{R}$ with characteristic measure $\lambda(\cdot)$. Denote by $N(d t, d u)$ the Poisson counting measure associated with $p$, i.e., $N(t, U)=\sum_{s \in D_{p}, s \leq t} I_{U}(p(s))$ for $U \in \mathcal{B}(\mathbb{R})$. Let $\tilde{N}(d t, d u):=N(d t, d u)-d t \lambda(d u)$ be the compensated Poisson measure associated with $N(d t, d u)$. In what follows, we further assume that $\int_{U}|u|^{p} \lambda(d u)<\infty$ for any $p \geq 2$.

In this section we consider SDDE with jumps on $\mathbb{R}^{n}$ :

$$
d X(t)=b(X(t), X(t-\tau)) d t+\int_{U} h(X(t), X(t-\tau), u) \tilde{N}(d t, d u), \quad t \in[0, T]
$$

with initial data $X(\theta)=\xi(\theta), \theta \in[-\tau, 0]$. We assume that

(A3) $b: \mathbb{R}^{n} \times \mathbb{R}^{n} \rightarrow \mathbb{R}^{n}$ satisfies assumption (A1);

(A4) $h: \mathbb{R}^{n} \times \mathbb{R}^{n} \times U \rightarrow \mathbb{R}^{n}$ and there exists $L_{3}>0$ such that

$$
\left|h\left(x_{1}, y_{1}, u\right)-h\left(x_{2}, y_{2}, u\right)\right| \leq\left(L_{3}\left|x_{1}-x_{2}\right|+V_{3}\left(y_{1}, y_{2}\right)\left|y_{1}-y_{2}\right|\right)|u|
$$

for $x_{i}, y_{i} \in \mathbb{R}^{n}, i=1,2$, and $u \in U$, where $V_{3}: \mathbb{R}^{n} \times \mathbb{R}^{n} \rightarrow \mathbb{R}_{+}$such that

$$
V_{3}(x, y) \leq K_{3}\left(1+|x|^{q_{3}}+|y|^{q_{3}}\right)
$$

for some $K_{3}>0, q_{3} \geq 1$ and arbitrary $x, y \in \mathbb{R}^{n}$. 
Remark 3.1. The jump coefficient may also be highly nonlinear with respect to the delay arguments; e.g., for $x, y \in \mathbb{R}, u \in U$ and $q>1, h(x, y, u)=y^{q} u$ satisfies (A4).

Fix $T>0$ and let the stepsize $\triangle$ be defined by (2.3). The EM scheme associated with (3.1) is defined as follows:

$$
d Y(t)=b(\tilde{Y}(t), \tilde{Y}(t-\tau)) d t+\int_{U} h(\tilde{Y}(t), \tilde{Y}(t-\tau), u) \tilde{N}(d t, d u),
$$

where $\bar{Y}(t):=Y(k \triangle)$ for $t \in[k \triangle,(k+1) \triangle), k=0,1, \cdots, M-1$, and $\bar{Y}(\theta):=$ $\xi(\theta), \theta \in[-\tau, 0]$.

To reveal the convergence order of the EM scheme (3.3), we need two auxiliary lemmas, where the first one is the Bichteler-Jacod inequality for Poisson integrals; see, e.g., [11, Lemma 3.1].

Lemma 3.2. Let $\Phi: \mathbb{R}_{+} \times U \rightarrow \mathbb{R}^{n}$ and assume that

$$
\int_{0}^{t} \int_{U} \mathbb{E}|\Phi(s, u)|^{p} \lambda(d u) d s<\infty, \quad t \geq 0, \quad p \geq 2 .
$$

Then there exists $D(p)>0$ such that

$$
\begin{aligned}
\mathbb{E}\left(\sup _{0 \leq s \leq t}\left|\int_{0}^{s} \int_{U} \Phi(r, u) \tilde{N}(d u, d s)\right|^{p}\right) \leq & D(p)\left\{\mathbb{E}\left(\int_{0}^{t} \int_{U}|\Phi(s, u)|^{2} \lambda(d u) d s\right)^{\frac{p}{2}}\right. \\
& \left.+\mathbb{E} \int_{0}^{t} \int_{U}|\Phi(s, u)|^{p} \lambda(d u) d s\right\} .
\end{aligned}
$$

Using the lemma above and the similar argument of Lemma 2.2, we have

Lemma 3.3. Let (A3) and (A4) hold. Then (3.1) has a unique global solution $(X(t))_{t \in[0, T]}$. Moreover, for any $p \geq 2$ there exists $C>0$ such that

$$
\mathbb{E}\left(\sup _{0 \leq t \leq T}|X(t)|^{p}\right) \vee \mathbb{E}\left(\sup _{0 \leq t \leq T}|Y(t)|^{p}\right) \leq C
$$

and

$$
\mathbb{E}|Y(t)-\bar{Y}(t)|^{p} \leq C \triangle .
$$

Remark 3.4. We remark that for $p \geq 2$ all $p$ th moments of $Y(t)-\bar{Y}(t)$ are bounded by $\triangle$ up to a constant, which is completely different from the Brownian motion case (2.6) . This is due to the fact that all moments of the increment $\tilde{N}((0,(i+$ $1) \triangle], d u)-\tilde{N}((0, i \triangle], d u)$ have order $O(\triangle)$ for $\triangle \in(0,1)$.

We now state our main result in this section.

Theorem 3.5. Let (A3) and (A4) hold. For any $p \geq 2$ and arbitrary $\theta, \alpha \in(0,1)$, there exists $C>0$, independent of $\triangle$, such that

$$
\mathbb{E}\left(\sup _{0 \leq s \leq T}|X(s)-Y(s)|^{p}\right) \leq C \triangle^{\frac{1}{(1+\theta)^{[T / \tau](1+\alpha)}}} .
$$


Proof. The proof of Theorem 3.5] is similar to that of Theorem 2.4, while we give a sketch of its argument to highlight the differences with the Brownian motion case. Set

$Z(t):=X(t)-Y(t), \quad \bar{Z}(t):=Y(t)-\bar{Y}(t), \quad \tilde{Z}(t):=(X(t), \bar{Y}(t)) \in \mathbb{R}^{2 n}, \quad t \in[0, T]$.

Define for $t \in[0, T]$,

$$
\Gamma_{1}(t):=b(X(t), X(t-\tau))-b(\bar{Y}(t), \bar{Y}(t-\tau))
$$

and

$$
\Gamma_{2}(t, u):=h(X(t), X(t-\tau), u)-h(\bar{Y}(t), \bar{Y}(t-\tau), u)
$$

For $V_{\delta \epsilon} \in C^{2}\left(\mathbb{R}^{n} ; \mathbb{R}_{+}\right)$, defined by (2.13), the Itô formula and the Taylor expansion give that for $t \in[0, T]$,

$$
\begin{aligned}
& V_{\delta \epsilon}(Z(t))=\int_{0}^{t}\left\langle\left(V_{\delta \epsilon}\right)_{x}(Z(s)), \Gamma_{1}(s)\right\rangle d s+\int_{0}^{t} \int_{U}\left\{V_{\delta \epsilon}\left(Z(s)+\Gamma_{2}(s, u)\right)\right. \\
& \left.\quad-V_{\delta \epsilon}(Z(s))-\left\langle\left(V_{\delta \epsilon}\right)_{x}(Z(s)), \Gamma_{2}(s, u)\right\rangle\right\} \lambda(d u) d s \\
& \quad+\int_{0}^{t} \int_{U}\left\{V_{\delta \epsilon}\left(Z(s)+\Gamma_{2}(s, u)\right)-V_{\delta \epsilon}(Z(s))\right\} \tilde{N}(d u, d s) \\
& =\int_{0}^{t}\left\langle\left(V_{\delta \epsilon}\right)_{x}(Z(s)), \Gamma_{1}(s)\right\rangle d s \\
& \quad+\int_{0}^{t} \int_{U}\left\{\int_{0}^{1}\left\langle\left(V_{\delta \epsilon}\right)_{x}\left(\theta \Gamma_{2}(s, u)+Z(s)\right)-\left(V_{\delta \epsilon}\right)_{x}(Z(s)), \Gamma_{2}(s, u)\right\rangle d \theta\right\} \lambda(d u) d s \\
& \quad+\int_{0}^{t} \int_{U}\left\{\int_{0}^{1}\left\langle\left(V_{\delta \epsilon}\right)_{x}\left(\theta \Gamma_{2}(s, u)+Z(s)\right), \Gamma_{2}(s, u)\right\rangle d \theta\right\} \tilde{N}(d u, d s) .
\end{aligned}
$$

Then by (3.6), together with (2.11) and (2.14), we deduce that

$$
\begin{aligned}
|Z(t)| \leq & \epsilon+V_{\delta \epsilon}(Z(t)) \\
\leq & \epsilon+\int_{0}^{t}\left|\Gamma_{1}(s)\right| d s+2 \int_{0}^{t} \int_{U}\left|\Gamma_{2}(s, u)\right| \lambda(d u) d s \\
& +\int_{0}^{t} \int_{U}\left\{\int_{0}^{1}\left\langle\left(V_{\delta \epsilon}\right)_{x}\left(\theta \Gamma_{2}(s, u)+Z(s)\right), \Gamma_{2}(s, u)\right\rangle d \theta\right\} \tilde{N}(d u, d s), \quad t \in[0, T] .
\end{aligned}
$$

Furthermore, note from (2.2), (3.2) and (3.4) that

$$
\mathbb{E}\left(\sup _{0 \leq t \leq T} V_{1}^{q}(\tilde{Z}(s-\tau))\right)+\mathbb{E}\left(\sup _{0 \leq t \leq T} V_{3}^{q}(\tilde{Z}(s-\tau))\right) \leq C, \quad q \geq 2
$$


Consequently, for any $p \geq 2$ and $t \in[0, T]$, using (2.14), (3.5), Lemma 3.2, the Hölder inequality, (A3) and (A4), we arrive at

$$
\begin{aligned}
\mathbb{E}\left(\sup _{0 \leq s \leq t}|Z(s)|^{p}\right) \leq & 2^{p-1}\left\{\epsilon^{p}+\mathbb{E}\left(\sup _{0 \leq s \leq t} V_{\delta \epsilon}^{p}(Z(s))\right)\right\} \\
\leq & C\left\{\epsilon^{p}+\int_{0}^{t} \mathbb{E}\left|\Gamma_{1}(s)\right|^{p} d s+\int_{0}^{t} \int_{U} \mathbb{E}\left|\Gamma_{2}(s, u)\right|^{p} \lambda(d u) d s\right. \\
& \left.+\mathbb{E}\left(\int_{0}^{t} \int_{U}\left|\Gamma_{2}(s, u)\right|^{2} \lambda(d u) d s\right)^{\frac{p}{2}}\right\} \\
\leq & C\left\{\epsilon^{p}+\int_{0}^{t} \mathbb{E}\left|\Gamma_{1}(s)\right|^{p} d s+\int_{0}^{t} \int_{U} \mathbb{E}\left|\Gamma_{2}(s, u)\right|^{p} \lambda(d u) d s\right\} \\
\leq & C\left\{\epsilon^{p}+\int_{0}^{t} \mathbb{E}(|X(s)-\bar{Y}(s)|\right. \\
& \left.+V_{1}(\tilde{Z}(s-\tau))|X(s-\tau)-\tilde{Y}(s-\tau)|\right)^{p} d s+\int_{0}^{t} \mathbb{E}(|X(s)-\bar{Y}(s)| \\
& \left.\left.+V_{3}(\tilde{Z}(s-\tau))|X(s-\tau)-\tilde{Y}(s-\tau)|\right)^{p} d s\right\} \\
\leq & C\left\{\epsilon^{p}+\triangle+\int_{0}^{t}\left\{\mathbb{E}|Z(s)|^{p}+\mathbb{E}\left(V_{1}^{p}(\tilde{Z}(s-\tau))|Z(s-\tau)|^{p}\right)\right.\right. \\
& +\mathbb{E}\left(V_{1}^{p}(\tilde{Z}(s-\tau))|\bar{Z}(s-\tau)|^{p}\right)+\mathbb{E}\left(V_{3}^{p}(\tilde{Z}(s-\tau))|Z(s-\tau)|^{p}\right) \\
& \left.\left.+\mathbb{E}\left(V_{3}^{p}(\tilde{Z}(s-\tau))|\bar{Z}(s-\tau)|^{p}\right)\right\} d s\right\} \\
\leq & C\left\{\epsilon^{p}+\triangle+\int_{0}^{t} \mathbb{E}|Z(s)|^{p} d s\right. \\
& \left.+\int_{0}^{t}\left\{\left(\mathbb{E}|Z(s-\tau)|^{p(1+\theta)}\right)^{\frac{1}{1+\theta}}+\left(\mathbb{E}|\bar{Z}(s-\tau)|^{p(1+\theta)}\right)^{\frac{1}{1+\theta}}\right\} d s\right\}
\end{aligned}
$$

where $\theta \in(0,1)$ is an arbitrary constant. Then an application of the Gronwall inequality gives that

$$
\begin{aligned}
\mathbb{E}\left(\sup _{0 \leq s \leq t}|Z(s)|^{p}\right) \leq & C\left\{\triangle+\int_{0}^{t}\left\{\left(\mathbb{E}|Z(s-\tau)|^{p(1+\theta)}\right)^{\frac{1}{1+\theta}}\right.\right. \\
& \left.\left.+\left(\mathbb{E}|\bar{Z}(s-\tau)|^{p(1+\theta)}\right)^{\frac{1}{1+\theta}}\right\} d s\right\}, \quad t \in[0, T],
\end{aligned}
$$

by taking $\epsilon=\triangle^{\frac{1}{p}}$. For $\theta \in(0,1)$ in (3.7) and any $\alpha \in(0,1)$, let

$$
p_{i}:=p(1+\theta)^{([T / \tau]+1-i)(1+\alpha)}, \quad i=1,2, \cdots,[T / \tau]+1 .
$$

It is trivial to see that

$$
(1+\theta) p_{i+1}<p_{i} \text { and } p_{[T / \tau]+1}=p, \quad i=1,2, \cdots,[T / \tau] .
$$

Noting that $Z(t)=\bar{Z}(t)=0$ for $t \in[-\tau, 0]$, by (3.7) we get

$$
\mathbb{E}\left(\sup _{0 \leq s \leq \tau}|Z(s)|^{p_{1}}\right) \leq C \triangle \text {. }
$$


This, together with (3.5), (3.7) and the Hölder inequality, yields that

$$
\begin{aligned}
\mathbb{E}\left(\sup _{0 \leq s \leq 2 \tau}|Z(s)|^{p_{2}}\right) & \leq C\left\{\triangle+\triangle^{\frac{1}{1+\theta}}+\int_{0}^{2 \tau}\left(\mathbb{E}|Z(s-\tau)|^{\left.p_{2}(1+\theta)\right)^{\frac{1}{1+\theta}}} d s\right\}\right. \\
& \leq C\left\{\triangle+\triangle^{\frac{1}{1+\theta}}+\int_{0}^{2 \tau}\left(\mathbb{E}|Z(s-\tau)|^{p_{1}}\right)^{\frac{p_{2}}{p_{1}}} d s\right\} \\
& \leq C\left\{\triangle+\triangle^{\frac{1}{1+\theta}}+\triangle^{\frac{p_{2}}{p_{1}}}\right\} \\
& \leq C \triangle^{\frac{p_{2}}{p_{1}}}
\end{aligned}
$$

where the last step is due to (3.8). Similarly, we obtain from (3.7)-(3.9) that

$$
\begin{aligned}
\mathbb{E}\left(\sup _{0 \leq s \leq 3 \tau}|Z(s)|^{p_{3}}\right) & \leq C\left\{\triangle+\triangle^{\frac{1}{1+\theta}}+\int_{0}^{3 \tau}\left(\mathbb{E}|Z(s-\tau)|^{p_{3}(1+\theta)}\right)^{\frac{1}{1+\theta}} d s\right\} \\
& \leq C\left\{\triangle+\triangle^{\frac{1}{1+\theta}}+\int_{0}^{3 \tau}\left(\mathbb{E}|Z(s-\tau)|^{p_{2}}\right)^{\frac{p_{3}}{p_{2}}} d s\right\} \\
& \leq C\left\{\triangle+\triangle^{\frac{1}{1+\theta}}+\triangle^{\frac{p_{3}}{p_{1}}}\right\} \\
& \leq C \triangle^{\frac{p_{3}}{p_{1}}}
\end{aligned}
$$

Following the previous procedures gives that

$$
\mathbb{E}\left(\sup _{0 \leq s \leq T}|Z(s)|^{p}\right) \leq C \triangle^{\frac{1}{(1+\theta)^{[T / \tau](1+\alpha)}}}
$$

and the proof is therefore complete.

Remark 3.6. By Theorem 3.5, with $p \geq 2$ increasing, the convergence order of the EM scheme (3.3) is decreasing, which is quite different from the Brownian motion case with a constant order $\frac{1}{2}$, and it is therefore best to use the meansquare convergence for the jump case. On the other hand, we reveal that the order of mean-square convergence is close to $\frac{1}{2}$, although the jump diffusion may be highly nonlinear with respect to the delay variables.

\section{REFERENCES}

1. J. Bao, B. Böttcher, X. Mao and C. Yuan, Convergence rate of numerical solutions to SFDEs with jumps, J. Comput. Appl. Math., 236 (2011), no. 2, 119-131. MR2827394

2. I. Gyöngy and M. Rásonyi, A note on Euler approximations for SDEs with Hölder continuous diffusion coefficients, Stochastic Process. Appl., 121 (2011), no. 10, 2189-2200. MR2822773

3. I. Gyöngy, A note on Euler's approximations, Potential Anal., 8 (1998), no. 3, 205-216. MR1625576 (99d:60060)

4. D. J. Higham and P. E. Kloeden, Numerical methods for nonlinear stochastic differential equations with jumps, Numer. Math., 101 (2005), no. 1, 101-119. MR2194720(2006k:65013)

5. D. J. Higham, X. Mao and A. M. Stuart, Strong convergence of Euler-type methods for nonlinear stochastic differential equations, SIAM J. Numer. Anal., 40 (2002), no. 3, 10411063. MR1949404 (2003j:65005)

6. Hu, Y., Semi-implicit Euler-Maruyama scheme for stiff stochastic equations, The Silivri Workshop, Progr. Probab. 38, H. Koerezlioglu, ed., Birkhäuser, Boston, 1996, 183-202. MR.1396331 (97e:60101)

7. N. Jacob, Y. Wang and C. Yuan, Numerical solutions of stochastic differential delay equations with jumps, Stoch. Anal. Appl., 27 (2009), no. 4, 825-853. MR2541379 (2010j:60144)

8. P. E. Kloeden and E. Platen, Numerical Solution of Stochastic Differential Equations, Springer, Berlin, 1992. MR1214374 (94b:60069) 
9. U. Küchler and E. Platen, Strong discrete time approximation of stochastic differential equations with time delay, Math. Comput. Simulation, 54 (2000), no. 1-3, 189-205. MR.1800113 (2001j:60111)

10. X. Mao and S. Sabanis, Numerical solutions of stochastic differential delay equations under local Lipschitz condition, J. Comput. Appl. Math., 151 (2003), no. 1, 215-227. MR.1950237 (2003k:65008)

11. C. Marinelli, C. Prévôt and M. Röckner, Regular dependence on initial data for stochastic evolution equations with multiplicative Poisson noise, J. Funct. Anal., 258 (2010), no. 2, 616-649. MR2557949(2011a:60230)

12. E. Platen and N. Bruti-Liberati, Numerical solution of stochastic differential equations with jumps in finance, Springer-Verlag, Berlin, 2010. MR2723480 (2012b:60001)

13. H. Schurz, Stability, stationarity, and boundedness of some implicit numerical methods for stochastic differential equations and applications, Logos Verlag Berlin, Berlin, 1997. MR.1991701

14. F. Wu, X. Mao and K. Chen, The Cox-Ingersoll-Ross model with delay and strong convergence of its Euler-Maruyama approximate solutions, Appl. Numer. Math., 59 (2009), no. 10, 26412658. MR2553159(2011a:91290)

15. C. Yuan and X. Mao, A note on the rate of convergence of the Euler-Maruyama method for stochastic differential equations, Stoch. Anal. Appl., 26 (2008), no. 2, 325-333. MR2399739 (2009b:65013)

Department of Mathematics, Swansea University, Singleton Park, Swansea, SA2 8PP, UNITED KINGDOM

E-mail address: majb@swansea.ac.uk

Department of Mathematics, Swansea University, Singleton Park, Swansea, SA2 8PP, United Kingdom

E-mail address: C.Yuan@swansea.ac.uk 